\title{
LEFT PARADUODENAL HERNIA AS A RARE CAUSE OF INTESTINAL OBSTRUCTION-LAPAROSCOPIC MANAGEMENT
}

KEY WORDS: Left Paraduodenal Hernia[PDH] Intestinal Obstruction, Laparoscopic Repair, CECT.

\section{Dr. Bhavesh Shelke*}

\section{Dr. Abhijit Joshi}

Resident in Dept. of General \& Laparoscopic Surgery DR L H Hiranandani Hospital, Hillside avenue, Hiranandani Gardens, Powai, Mumbai-400076, India *Corresponding Author

MBBS, MS (Gen Surgery), Fellowship in Laparoscopy, Diploma in Advanced Laparoscopy, Consultant General, G I \& Endo-Laparoscopic Surgeon.

We report a case of 33 years old male who presented with colicky pain in left upper abdomen on and off since 15 days, one episode of bilious vomiting and abdominal distension with constipation since 2 days. The case of intestinal obstruction was diagnosed as a Left Paraduodenal Hernia[PDH] on contrast enhanced computed tomography of the abdomen(CECT) and managed with laparoscopic repair. Through this case report, we want to give the message that PDH though rare should be considered as a differential diagnosis in a case of intestinal obstruction and can be managed successfully with laparoscopic approach even in an emergency situation.

\section{INTRODUCTION:}

Intestinal obstruction due to internal hernia is very rare. Among internal hernias PDH is very common[1]. Traditionally it has been treated with open surgery, but since the advent of minimal access surgery, laparoscopy is an additional valuable therapeutic tool.

\section{CASE REPORT:}

A 33 years old male patient presented to the emergency ward with colicky pain in left upper abdomen on and off since 15 days, one episode of bilious vomiting and abdominal distension with constipation since 2 days. On examination, his vitals were stable. On per abdominal examination, he had a distended abdomen, tender left hypochondrium and hyperperistalsis in the central abdomen. A per rectal examination revealed an empty rectum. Laboratory reports were unremarkable. X ray abdomen done in standing position showed distended small bowel loops and few air-fluid levels in the left upper abdomen. Ultrasonography of abdomen revealed vague probe tenderness, matted appearance of small bowel loops, pseudokidney appearance and increased omental fat echogenicity. A CECT scan of the abdomen and pelvis showed multiple contrast filled small bowel loops behind the descending mesocolon suggestive of Left PDH. The patient underwent laparoscopic repair of left PDH. During laparoscopy, most of the small bowel loops were seen entering a recess behind descending colon. Gentle attempts at reduction yielded favourable results and most of the entrapped small bowel could be reduced back into the generalised peritoneal cavity. The anterior border of the narrow inlet to the fossa which contained the inferior mesenteric vein [IMV] was then divided using ultrasonic shears. This wide division of the neck of the sac thus marsupialised the recess i.e. the fossa of Landzert and merged it with the peritoneal cavity. The post-operative recovery of the patient was uneventful. On the third postoperative day, he was discharged and till present day, remains asymptomatic.

\section{DISCUSSION:}

Internal hernias arise due to abnormal protrusion of viscera through an abnormal opening in the mesentery or peritoneum within the boundaries of abdominal cavity [4].

Paraduodenal hernias are the most common type of internal hernias[53\%][1].

Para-duodenal hernia is defined as abnormal protrusion of viscera into the para-duodenal fossa and results in less than $1 \%$ of all cases of small intestinal obstruction[1].
Left PDH [75\%] is commoner than right PDH [25\%] [1].Embryological development of right para-duodenal hernia consists of non-rotation of pre-arterial segment of midgut [2], whereas development of left PDH consists of normal rotation of gut but abnormal fixation of the descending mesocolon to the posterior abdominal wall, which leads to formation of an unsupported area[Landzert's fossa] and herniation of contents into it. This lies lateral to the fourth part of the duodenum, posterior to the inferior mesenteric vein [IMV], the ascending branch of the left colic artery [LCA] and anterior to posterior parietal peritoneum [2].

It may present i) with features of bowel obstruction, ii) as a lump in the abdomen, iii) as an accidental discovery during another operation or iv) incidentally during imaging, showing small bowel loops to the left of the midline [2].

A CECT abdomen is the diagnostic clincher. CECT abdomen of left PDH shows clumped small bowel loops between stomach or transverse and descending colon anteriorly and pancreas or left adrenal gland posteriorly. Inferior mesenteric vein and ascending branch of left colic artery lie in the anterior or medial border of the sac [4].

Treatment of left PDH consists of surgical repair of hernia.The procedure consists of $\alpha$ )reduction of bowel contents and then b)repair of the defect. The repair of the defect entails either i) suture closure/mesh repair or ii) marsupialisation of the recess and it's merging with the peritoneal cavity. While performing marsupialisation, IMV can be clipped and divided in order to widen the neck of the sac. Mesh repair is reserved for larger defects and recurrent hernias[5].

Laparoscopy has the advantage of minimal invasion, magnified view, lesser post-operative morbidity, shorter hospital stay and early recovery [3].

\section{CONCLUSION:}

In a case of intestinal obstruction; PDH, though a rare entity, should be considered as a differential diagnosis. CECT abdomen is diagnostic of PDH, with high specificity. Even in emergency presentations, PDH can be managed successfully with laparoscopy.

\section{FUNDING: None}

CONFLICT OF INTEREST: There is no conflict of interest. List of abbreviations:

CECT: Contrast Enhanced Computerized Tomography Scan PDH: Para-duodenal Hernia

Fig: Figure 


\section{LCA: Left Colic Artery}

IMV:Inferior Mesenteric Vein

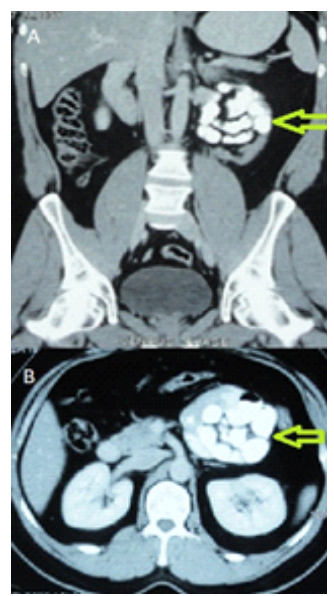

Fig.1CECT Abdomen+Pelvis

A] CORONAL view showing clumped small bowel loops to the left of midline

B] AXIAL view showing clumped small bowel loops behind descending colon

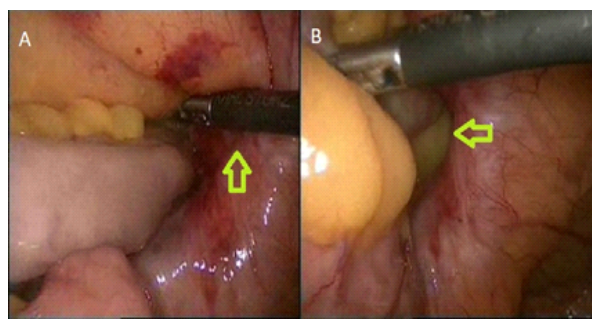

Fig.2 A] Small bowel loops entering into the Landzert's fossa B] Small bowel loops being delivered out of Landzert's fossa.

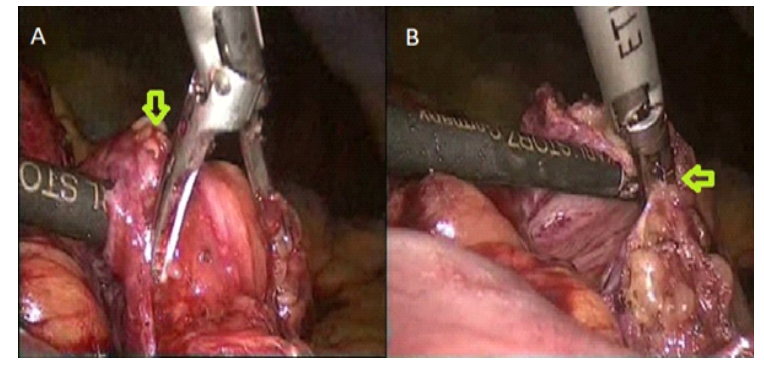

Fig. 3 Anterior border of inlet to Landzert's fossa being lifted up \& presented to the ultrasonic shears B] Anterior border of inlet to Landzert's fossa being divided

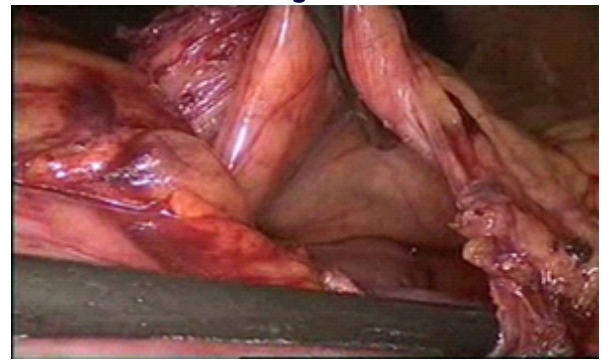

Fig.4 Whole Fossa of Landzert merged with peritoneal cavity

\section{REFERENCES:}

1. Rahaf S, Huda S, Yazeed B, Haifaa M. Rare Case of Small Bowel Obstruction Due to Paraduodenal Hernia Am J Case Rep. 2019; 20: 1581-1586 doi: 10.12659/AJCR.918403

2. Bartlett $M$, Wang $C$, Williams W. The surgical management of paraduodenal hernia. Ann Surg 1968; 168:249-54.

3. Shadhu K, Ramlagun D, Ping X. Para-duodenal hernia: a report of five cases and review of literature. BMC Surg 18, 32 [2018]. https:// doi. org/ 10.1186/s l

\section{3-018-0365-8}

4. Martin L, Merkle E, Thompson W. Review of internal hernias: radiographic and clinical findings. AJR Am J Roentgenol. 2006:186 (3): 703-17. doi:10.2214/AJR.05.0644

5. Parmar B, Parmar R. Laparoscopic management of left paraduodenal hernia. J Min Access Surg 2010 [cited 2020 May 21];6:122-4. 